\title{
Incidencia de la procrastinación en la autoeficacia académica de estudiantes en tiempos de COVID-19
}

\author{
Incidence of procrastination on the academic self-efficacy of students in times of COVID-19 \\ Incidência de procrastinação na autoeficácia acadêmica de alunos em tempos de COVID-19
}
Jessica Karina Carbajal Villanueva
jekacarbajalvillanueva@gmail.com
https://orcid.org/0000-0001-9395-0838
Universidad César Vallejo, Lima-Perú

\author{
Violeta Cadenillas Albornoz \\ cadealbo@ucvvirtual.edu.pe \\ https://orcid.org/0000-0002-4526-2309 \\ Universidad César Vallejo, Lima-Perú
}

\section{RESUMEN}

La procrastinación es una conducta negativa en la cual los estudiantes postergan la realización de sus actividades académicas, porque se sienten desmotivados o estresados, esta condición se puede deber a múltiples factores; como fue la pandemia del COVID-19, por el cambio de clases a distancia o virtuales, adaptarse a este nuevo sistema educativo afectó su rendimiento académico. Se formuló como objetivo demostrar la incidencia de la procrastinación en la autoeficacia académica en estudiantes de educación secundaria en tiempos de COVID-19. Se empleó como metodología el enfoque cuantitativo, con diseño explicativo descriptivo, a un amuestra de 169 estudiante del VII ciclo del nivel secundario en una institución privada. Los resultados obtenidos del procesamiento estadístico permitieron se concluyó que, existe incidencia significativa entre la procrastinación y la autoeficacia académica, el desgano y desmotivación estuvieron influenciados por el contexto de la pandemia originada por el COVID-19.

Palabras clave: Autoeficacia; COVID-19; Estudiantes; Procrastinación

ABSTRACT

RESUMO

Procrastination is a negative behavior in which students postpone the completion of their academic activities, because they feel unmotivated or stressed, this condition may be due to multiple factors; as was the pandemic of COVID-19, due to the change of distance or virtual classes, adapting to this new educational system affected their academic performance. The objective of this study was to demonstrate the incidence of procrastination on academic self-efficacy in high school students in times of COVID-19. A quantitative approach was used as methodology, with a descriptive explanatory design, to a sample of 169 students of the VII cycle of the secondary level in a private institution. The results obtained from the statistical processing allowed us to conclude that there is a significant incidence between procrastination and academic self-efficacy, and that the lack of motivation and motivation were influenced by the context of the pandemic caused by COVID- 19 .

Key words: Self-efficacy; COVID-19; Students; Procrastination
A procrastinação é um comportamento negativo no qual os estudantes adiam a conclusão de suas atividades acadêmicas, porque se sentem desmotivados ou estressados, esta condição pode ser devida a múltiplos fatores; assim como a pandemia da COVID-19, devido à mudança das aulas à distância ou virtuais, a adaptação a este novo sistema educacional afetou seu desempenho acadêmico. $O$ objetivo deste estudo era demonstrar o impacto da procrastinação na auto-eficácia acadêmica dos alunos do ensino médio em tempos de COVID-19. A metodologia utilizada foi uma abordagem quantitativa, com um desenho explicativo descritivo, com uma amostra de 169 estudantes do sétimo ciclo do ensino médio em uma instituição privada. Os resultados obtidos com o processamento estatístico nos permitiram concluir que há uma incidência significativa entre procrastinação e auto-eficácia acadêmica, e que a falta de entusiasmo e a desmotivação foram influenciadas pelo contexto da pandemia causada pela COVID-19.

Palavras-chave: Auto-eficácia; COVID-19; Estudantes; Procrastinação 


\section{INTRODUCCIÓN}

El año 2020 el gobierno peruano estableció que, en los colegios, institutos y universidades, se desarrollaran las clases remotas, a distancia o virtuales, debido al emergencia sanitaria, causada por el virus del COVID-19, este contexto originó cambios abruptos en el sistema educativo que repercutieron en los estudiantes al escolarizarse sus hogares; esta situación develó dificultades las cuales los afectaron, como fueron las brechas de conectividad al no poder acceder a internet que les permitieran mantener comunicación sincrónica y asincrónica con sus docentes (Gómez y Escobar, 2021) limitaciones en el empleó de la competencias digitales (Levan etal.,2019) y afecciones emocionales provocadas por el encierro, generándoles estrés, desgano e irritabilidad (Sánchez, 2021).

Resultó complicado al principio adaptarse la educación remota o virtual, a los estudiantes les costó realizar acciones sencillas como compartir sus evidencias, emplear las plataformas o asistir a las videoconferencias; entre las razones que ocasionaron estas complicaciones estuvieron no contar con internet y los recursos tecnológicos necesario, porque no estaban preparados para este cambio; el dispositivo más empleado para poder llevar las clases virtuales fueron los equipos celulares mediante el WhatsApp, donde se entregaban imágenes, documentos o videos (Rodríguez y Formoso, 2020); además fue la herramienta más económica a la cual se podía acceder (Vásquez et al., 2020), también fueron utilizadas las tabletas y computadoras, sin embargo, esta solución nos les brindó mucha comodidad generándoles estrés y desmotivación, dando resultados negativos como fue bajar su rendimiento académico o desinterés por el estudio.
No hay duda que implementar el nuevo sistema educativo, fue un factor determinante para que muchos estudiantes no se sintiera satisfechos y con ganas de desarrollar las actividades de extensión recomendadas por los docentes; ensayaron múltiples argumentos, como el no tener internet, recursos tecnológicos o justificaciones falsas, con tal de evadir su responsabilidad académica, por tal razón fue necesario comprender la procrastinación (Carranza y Ramírez, 2013) es la postergación en realizar las tareas priorizando actividades recreativas las cuales brindan mayor satisfacción, este aplazamiento se desencadena por factores subjetivos (Dominguez y Lara, 2014) también toma en cuenta la autorregulación de las personas para dosificar sus tiempos que les permita desarrollar trabajos eficientes (Rebeteza, et al., 2016), emocionalmente la procrastinacción genera stress, ansiedad, culpa o depresión, la persona se siente agobiada al no poder hacer las actividades para cuando son requeridas o no gestionar correctamente los tiempos (Devi y Dhull, 2017).

La evaluación formativa la cual es sistemática, continua y flexible, donde todo aporte que realicé el estudiante sea de manera sincrónica o asincrónica es valorado, por tanto, las actividades de extensión llamadas antes tareas consideradas evidencias son productivas cuando se demuestra sus progresos o logros de aprendizaje, teniendo en cuenta las competencias y capacidades requeridas, además, de acuerdo a la RV Nº 093 "Orientaciones pedagógicas para el servicio educativo de Educación Básica durante el año 2020 en el marco de la emergencia sanitaria por el Coronavirus COVID-19". (MINEDU, 2020), se priorizaron temas para ser trabajados en las instituciones educativas, provocando el 
conformismo y desapegó de algunos estudiantes, justificando sino le exigen, porque apurarse o dar más de sí al realizar una actividad, esta situación se complicó más cuando el Ministerio de Educación implementó la promoción guiada, en la cual todos los estudiantes a pesar de tener dificultades académicas; fueron promovidos al siguiente año, este contexto contribuyó a incrementar el desinterés de algunos estudiantes en practicar conductas de aprendizaje responsables.

De acuerdo a lo antes expuesto, la procrastinación además de provocar el bajo rendimiento académico, también influye en los estudiantes decidan abandonar la escuela, sentirse víctimas de afecciones psicológicas y estrés (Barraza y Barraza, 2019), detectar estas conductas negativas debe ser una prioridad en las instituciones educativas para atenderlas e involucrar a las familias como aliados. (Domínguez-Lara y Campos, 2017). En el contexto de la emergencia sanitaria los padres de familia presentaron limitaciones para acompañar a sus hijos en las clases a distancia (Janampa et al., 2021) por tal motivo, resultó complicado darle atención a esta conducta negativa de procrastinar.

Una forma de hacer frente a la procrastinación, puede ser motivar en los estudiantes conductas positivas autoformativas, en el Currículo Nacional de la Educación Básica (CNEB) (MINEDU, 2016) propone desarrollar procesos de aprendizaje autónomos, en los cuales sean capaces de gestionar la información haciendo uso de sus competencias y capacidades, esto es posible con la autoeficacia académica, ellos pueden regular la adquisición de conocimientos y reflexionar cómo los obtienen, para conseguir sus logos (Galleguillos y Olmedo, 2017; Rossi, et al., 2020).
En la autoeficacia es importante que cada individuo se analice y reconozca cuáles son sus fortalezas y habilidades, al reconocer las destrezas también influye la motivación (Cook y Artino, 2016) el estudiante motivado asume retos que al realizarlos siente satisfacción, CovarrubiasApablaza et al. (2019)sostuvo que en la autoeficacia se puede reconocer tres elementos: conductual, cognitivo y emocional; se pudo dimensionar en generalización, como la capacidad de dominar los cambios tiempos (Galleguillos, 2017 la segunda el nivel de dificultad en función a los retos y problemas, de toda adversidad se obtiene una oportunidad para mejorar y reinventarse; por último, la tercera dimensión es fortaleza, conforme una apersona socializa e interactúa con otros descubre cuáles son sus potencialidades y aptitudes. las realiza con satisfacción obteniendo buenos resultados, será su propia marca personal.

En el contexto de la emergencia sanitaria; no todo fue negativo porque existieron quienes se adaptaron a la nueva normalidad; en el aspecto educativo la resiliencia fue la clave para seguir adelante, encontrando en la educación a distancia o virtual una forma de adquirir nuevos conocimientos, emplear mejor la información que el acceso a internet ofreció y fortalecer las competencias digitales; es en este contexto que los estudiantes desarrollaron conductas proactivas empleado la autoeficacia como una forma de mejorar su desarrollo académico.

Este estudio plantea demostrar la incidencia de la procrastinación en la autoeficacia académica en estudiantes de educación secundaria en tiempos de COVID-19. El propósito es estudiar y observar el contexto de los estudiantes del nivel secundario, los 
cuales se encuentran en proceso de cambios y han sido afectados en su normal desarrollo académico con la educación a distancia o remota, se pudieron dar conductas negativas como la porcrastinación y en contra peso aptitudes positivas con la autoeficacia, por tal razón este estudió ofrece a la comunidad académica la percepción de los estudiantes en estos dos temas de estudio dentro del contexto que se dio el confinamiento obligatorio.

\section{MÉTODO}

El análisis del estudio se llevó a cabo mediante el enfoque cuantitativo, bajo el diseño descriptivo explicativo, para la recolección de la información se construyeron dos instrumentos bajo la escala de Likert validados por juicio de expertos, para mediar las variables procrastinación y eficacia académica. De acuerdo a los resultados que se muestran en la Tabla 1 empleando el Alfa de Cronbach, los dos instrumentos fueron confiables.

Tabla 1. Confiabilidad de los instrumentos.

\begin{tabular}{lccc}
\hline \multicolumn{1}{c}{ Variable } & Estadístico de Confiabilidad & Valor & N $^{\circ}$ de elementos \\
\hline Procrastinación & Alfa de Cronbach & 0.882 & 41 \\
Autoeficacia académica & Alfa de Cronbach & 0.903 & 18 \\
\hline
\end{tabular}

Los instrumentos fueron aplicados a una muestra de 169 estudiantes de Educación Básica Regular (EBR) pertenecientes al VII ciclo (Tercero, Cuarto y Quinto de Secundaria), cuyas edades fluctuaron entre los 15 y 17 años, por ser menores de edad se solicitó permiso a los padres de familia mediante un consentimiento informado, la distribución se realizó mediante formulario de Google drive.

Se tomaron en cuenta como criterios deinclusión que todos los estudiantes sean pertenecientes a la misma institución educativa y contra con matricula vigente en el Sistema de Información de Apoyo a la Gestión de la Institución Educativa (SIAGIE), los criterios de exclusión fueron desestimar a los estudiantes de otros ciclos y cuyos padres no hubieran aceptado dar su consentimiento.

\section{RESULTADOS Y DISCUSIÓN}

De acuerdo al procesamiento estadístico se obtuvieron los siguientes resultados.

De acuerdo a los datos que se muestran en la Tabla 2 se puede observar en la estadística descriptiva que los resultados de las dimensiones de la autoeficacia se encuentran mayoritariamente en el nivel medio, solo la generalización alcanzó alto con un $35.3 \%$; esto permitió inferir que lelemente existe deficiencias en los estudiantes para conseguir la autoeficacia, puede ser uno de los factores que influye la educación a distancia o virtual. 
Tabla 2. Niveles de las dimensiones de la variable autoeficacia académica.

\begin{tabular}{lcccccc}
\hline & Generalización & \multicolumn{2}{c}{ Nivel de dificultad } & \multicolumn{2}{c}{ Fortaleza } \\
& $\mathrm{F}$ & $\%$ & $\mathrm{f}$ & $\%$ & $\mathrm{~F}$ & $\%$ \\
\hline Bajo & 2 &, 7 & 8 & 2,8 & 7 & 2,5 \\
Medio & 67 & 23,7 & 80 & 28,3 & 92 & 32,5 \\
Alto & 100 & 35,3 & 81 & 28,6 & 70 & 24,7 \\
Total & $\mathbf{1 6 9}$ & $\mathbf{5 9 , 7}$ & $\mathbf{1 6 9}$ & $\mathbf{5 9 , 7}$ & $\mathbf{1 6 9}$ & $\mathbf{5 9 , 7}$ \\
\hline
\end{tabular}

Seguimente en la Tabla 3 se evidenció que de acuerdo a los modelos logísticos, resultados significativos $\left(X^{2}=99,339 ; \mathrm{p}<, 05\right)$, evidenciando que la procrastinación incide significativamente en la auto eficacia.

Tabla 3. Información sobre el ajuste del modelo que explica la incidencia de la procrastinación en la autoeficacia académica.

\begin{tabular}{lccccccc}
\hline \multicolumn{1}{c}{ Modelo } & $\begin{array}{c}\text { Información de ajuste de los modelos } \\
\text { Logaritmo de la } \\
\text { verosimilitud -2 }\end{array}$ & Chi-cuadrado & gl & Sig. & Cox y Snell &, 444 \\
\hline Sólo intersección & & & & & Nagelkerke &, 560 \\
Final &, 000 & 99,339 & 8 &, 000 & McFadden &, 373 \\
Función de enlace: Logit. & & & & & \\
\hline
\end{tabular}

En la Tabla 4 se observa que la Desviación $\left(x^{2}=15,057\right)$ muestran un $\mathrm{p}>, 05$; por tanto, se puede señalar que el modelo de regresión fue valido al considerar que la procrastinación incide en la autoeficacia académica.

Tabla 4. Bondad de ajuste del modelo que explica la incidencia de la procrastinación en la autoeficacia académica.

\begin{tabular}{lccc}
\hline & \multicolumn{2}{c}{ Bondad de ajuste } & \\
& Chi-cuadrado & Gl & Sig. \\
\hline Pearson & 12,930 & 30 &, 997 \\
Desvianza & 15,057 & 30 &, 989 \\
Función de enlace: Logit. & & & \\
\hline
\end{tabular}

En la Tabla 5, se muestran los resultados de la estimación de parámetros entre las variables de procrastinación y autoeficacia académica, lo cual se asumen los valores del coeficiente de Wald para la comparación y el valor de significación estadística mediante la regresión logística se evidenció la incidencia significativa entre ambas variables. 
Tabla 5. Estimaciones del parámetro para explicar que la procrastinación incide en la autoeficacia académica.

\begin{tabular}{|c|c|c|c|c|c|c|c|c|}
\hline \multicolumn{9}{|c|}{ Estimaciones de parámetro } \\
\hline & & \multirow{2}{*}{ Estimación } & \multirow{2}{*}{$\begin{array}{l}\text { Desv. } \\
\text { Error }\end{array}$} & \multirow{2}{*}{ Wald } & \multirow{2}{*}{ Gl } & \multirow{2}{*}{ Sig } & \multicolumn{2}{|c|}{$\begin{array}{c}\text { Intervalo de } \\
\text { confianza al 95\% }\end{array}$} \\
\hline & & & & & & & $\begin{array}{l}\text { Límite } \\
\text { inferior }\end{array}$ & $\begin{array}{l}\text { Límite } \\
\text { superior }\end{array}$ \\
\hline \multirow[t]{2}{*}{ Umbral } & [A. Acad= bajo $]$ & 15,885 & 469,535 & 001 & 1 & ,973 & $-904,386$ & 936,157 \\
\hline & [A. Acad = medio $]$ & 20,041 & 469,536 & ,002 & 1 & ,966 & $-900,234$ & 940,316 \\
\hline \multirow[t]{12}{*}{ Ubicación } & [P crónico emocional=bajo] & 34,199 & 671,711 & 003 & 1 & ,959 & $-1282,331$ & 1350,728 \\
\hline & [P crónico emocional = moderado $]$ & 32,995 & 671,711 & ,002 & 1 & ,961 & $-1283,533$ & 1349,524 \\
\hline & [P crónico emocional =alto $]$ & $0 \mathrm{a}$ & · & . & 0 & . & . & \\
\hline & [P estímulo demandante $=$ bajo $]$ & $-16,704$ & 480,348 & 001 & 1 & ,972 & $-958,168$ & 924,761 \\
\hline & [P estímulo demandante $=$ moderado $]$ & $-17,023$ & 480,348 & ,001 & 1 & ,972 & $-958,487$ & 924,441 \\
\hline & [P estímulo demandante $=$ alto $]$ & $0 \mathrm{a}$ & . & . & 0 & . & . & . \\
\hline & [P incomp. personal= bajo $]$ & 4,510 & 1,108 & 16,556 & 1 &, 000 & 2,338 & 6,683 \\
\hline & [P incomp. personal= moderado $]$ & 3,398 & 1,073 & 10,030 & 1 &, 002 & 1,295 & 5,501 \\
\hline & [P incomp. Personal= alto $]$ & $0 \mathrm{a}$ & · & . & 0 & . & . & • \\
\hline & [P aversión a la tarea $=$ bajo $]$ & ,803 & 1,548 & ,269 & 1 & ,604 & $-2,231$ & 3,836 \\
\hline & {$[\mathrm{P}$ aversión a la tarea $=$ moderado $]$} &, 184 & 1,447 &, 016 & 1 & 899 & $-2,652$ & 3,020 \\
\hline & [P aversión a la tarea $=$ alto $]$ & $0 \mathrm{a}$ & . & . & 0 & & . & \\
\hline
\end{tabular}

Función de enlace: Logit.

a. Este parámetro está establecido en cero porque es redundante.

\section{Discusión}

De acuerdo a los resultados obtenidos se demostró que efectivamente existió incidencia entre la procrastinación y la eficacia académica; sin embargo, los valores alcanzados en las dimensiones de la segunda variable se encontraron un tanto equilibrados para el nivel medio, lo ideal hubiera sido obtenido el nivel alto; como se ha descrito, este estudio se realizó en el contexto de la emergencia sanitaria, son múltiples los factores que afectan externos producto del nuevo sistema evacuativo, los cuales afectan a los estudiantes de secundaria con respecto a su rendimiento académico, los hallazgos coincidieron con los resultados de Estrada (2020) encontró una relación inversa y significativa entre las variables $(r s=-0,221 ; p=0,012<0,05)$ afirmando que los bajos niveles de autoeficacia se relaciona con altos niveles de procrastinación observados en los estudiantes.

En esta línea Burgos y Salas (2020) demostraron una correlación entre ambas variables; sin embargo en una de sus dimensione postergación y a utoeficacia; los autores infirieron que esto se debió porque a los estudiantes les faltó conocer sus habilidades y regular sus tiempos; por otro lado, Aspée et al., (2021) brindaron un 
modelo de correspondencia funcional entre la variable procrastinación académica y la variable compromiso, encontraron una relación inversa en la cual ambas variables no pueden ser necesariamente contradictorias.

La procrastinación también puede producir angustia $y$ ansiedad, porque al no saber priorizar sus actividades académicas se pueden sentir abrumados o en condiciones en las cuales serán evaluados les genera estrés y procrastinan, postergando sus responsabilidades, así lo establecieron Estremadoiro y Schulmeyer (2021) quienes hallaron un nivel medio de procrastinación entre los estudiantes, por consiguiente un ambiente adecuado o entorno favorable en el cual se encuentran los estudiantes satisfacción social ayuda a obtener un buen rendimiento académico y alejarse de conductas negativas (Gonzales et al., 2020) no hay duda que la pandemia ha alterado el ambiente físico einteracción social teniendo los estudiantes de educación secundarias conductas de procratinación; las cuales deberán ser superadas para el periodo lectivo 2022; porque es necesario que se acostumbren a la nueva normalidad.

\section{CONCLUSIONES}

Se concluyó que efectivamente existe incidencia entre la procarstinación y la eficacia académico en estudiantes de educación secundaria de una institución educativa privada, en tiempos de pandemia, los agentes externos sin son un factor importante para este resultado y estos son la situación singular que ha significado en el desarrollo del sistema educativo peruano la instauración de las clases a distancia o virtuales, producto del confinamiento obligatorio por el COVID-19, los estudiantes de VII ciclo quienes fueron objeto de este estudio, se encuentran en la última etapa de su formación escolar, por lo cual ya debieron haber adquirido aptitudes positivas al emplear sus competencias y capacidades para fortalecer sus experiencias de aprendizaje; sin embargo, el contexto de los efectos emocionales, conectividad y limitaciones en el uso de recursos tecnológicos fueron un factor determinante para el desgano, el año 2020 cuando estalló la emergencia sanitaria a los estudiantes de quinto, quienes estaban a punto de egresar, se les llamó la promoción pandemia producto del contexto que se vivió; sin embargo el año 2021 ya se debieron haber superado las dificultades iniciales y adoptar actitudes residentes en las cuales puedan adaptarse a la nueva normalidad; otro aspecto a tomar en consideración, es que los estudiantes extrañen las clases presenciales, es lamentable que el Perú sea uno de los últimos países en retornar a la prespecialidad la cual será efectiva en el periodo lectivo 2022.

Finalmente, este estudio dejó como reflexión que es imprescindible implementar desde el Departamento de Convivencia en las escuelas planes de mejora para fortalecer la predisposición en los estudiantes a los estudios.

\section{REFERENCIAS}

Aspée, J., González, J. y Herrera, Y. (2021). Relación funcional entre procrastinación académica y compromiso en estudiantes de educación superior: una propuesta de análisis. Perspectiva Educacional, 60(1), 4-22. https://dx.doi. org/10.4151/07189729-vol.60-iss.1-art.1116

Barraza Macías, A., y Barraza Nevárez, S. (2019). Procrastinación y estrés. Análisis de su relación en alumnos de educación media superior. CPU-e, Revista de Investigación Educativa, 28, 132-151. https://doi.org/10.25009/cpue. v0i28.2602 
Burgos-Torre, K. y Salas-Blas, E. (2020). Procrastinación y Autoeficacia académica en estudiantes universitarios limeños. Propósitos $y$ Representaciones, 8(3), e790. http://dx.doi. org/10.20511/pyr2020.v8n3.790

Cardoso, E., Cortés, J., y Cerecedo, M. (2020). Autoeficacia académica del alumnado de los posgrados en administración en tiempos del COVID-19. Propósitos y Representaciones, 8 (SPE3), e567. Doi: http://dx.doi.org/10.20511/ pyr2020.v8nSPE3.567

Carranza, R. y Ramírez, A. (2013). Procrastinación y características demográficas asociados en estudiantes universitarios. Procrastinación y Características Demográficas Asociados En Estudiantes Universitarios, 2(2), 95-108. https:// doi.org/10.17162/au.v0i2.284

Cook, D. y Artino Jr. (2016). Motivation to learn: An overview of contemporary theories. Medical Education, 50(10), 997-1014. https://doi: 10.1111/medu.13074

Covarrubias-Apablaza, C., Acosta-Antognoni, H., y Mendoza-Lira, M. (2019). Self-regulation learning and general self-efficacy and their relation with academic goals in university students. Formacion Universitaria, 12(6), 103-114. https://doi.org/10.4067/S071850062019000600103

Devi, R.y Dhull,P.(2017). Procrastination:abehavior need to be changed to get success. Internacional Education y Research Journal, 3(5), 475476. https://issuu.com/thewriterspublication/ docs/151-rajesh_devi

Dominguez-Lara, S. y Campos-Uscanga, Y. (2017). Influencia de la satisfacción con los estudios sobre la procrastinación académica en estudiantes de psicología: un estudio preliminar. Liber [online], 23(1), 123-135. ISSN 1729-4827. http://dx.doi.org/https://doi.org/10.24265/ liberabit. 2017.v23n1.09

Domínguez-Lara, S., Villegas, G. y Centeno, S. (2014). Procrastinación académica: validación de una escala en una muestra de estudiantes de una universidad privada. Liberabit, 20 (2), 293304
Estrada, E. (2020). Autoeficacia y procrastinación académica en estudiantes del séptimo ciclo de educación básica regular. Revista Horizonte de la Ciencia. https://doi.org/10.26490/uncp. horizonteciencia.2021.20.777

Estremadoiro B., y Schulmeyer M. (2021). Procrastinación académica en estudiantes universitarios. Revista Aportes de la Comunicación y la Cultura, (30), 51-66. Recuperado en 17 de noviembre de 2021, de http://www.scielo.org.bo/scielo.php?script=sci_ arttext\&pid=S2306-86712021000100004\&lng= es\&tlng=es

Galleguillos, P. y Olmedo, E. (2017). Autoeficacia académica y rendimiento escolar: un estudio metodológico y correlacional en escolares. ReiDoCrea, 6, 156- 169

Gómez, A, y Escobar, M. (2021). Educación virtual en tiempos de pandemia: Incremento de la desigualdad social en el Perú. Biblioteca Electrónica Científica En Línea, I, 1-13. https:// preprints.scielo.org/index.php/scielo/preprint/ view/1996\#.YFvtuoYYVe0.mendeley

Janampa, E., Gonzales-Sánchez, A., Bayona, G., y Valqui, J. (2021). Acompanhamento familiar aos alunos durante a pandemia COVID-19.pdf. Laplage Em Revista. https://doi.org/https://doi. org/10.24115/S2446-6220202173C1502p.43-50

Levan, L., Diaz, S., Guillen, P., Tello, S., Herrera, N., y Collantes, Z. (2019). Digital competences in education. Propositos y Representaciones, 7(2), 569-588

MINEDU. (2016). Currículo Nacional de Educación Básica Regular

MINEDU. (2020). $N^{\circ} 093$ RV Aprobar el documento normativo denominado "Orientaciones pedagógicas para el servicio educativo de Educación Básica durante el año 2020 en el marco de la emergencia sanitaria por el Coronavirus COVID-19”. https://www.gob.pe/institucion/ minedu/normas-legales/535987-093-2020minedu

Rebeteza, M.; Rochata, L.; Barsicsa, C. y Van der Linden, M. (junio, 2016). Procrastinationas a self-regulation failure: The role of inhibition, 
negative affect, and gender. Personatity and Individual Differences, 101, 435-439

Rodríguez Muñoz, R., y Formoso Mieres, A. A. (2020). Efectos de YouTube y WhatsApp en procesos de enseñanza - aprendizaje ante el nuevo coronavirus. Conrado, 16(77), 346-353

Rossi, T., Trevisol, A., Dos Santos-Nunes, D., Dapieve-Patias, N. y Von Hohendorff, J. (2020). Autoeficácia geral per-cebida e motivação para aprender em adolescentes do Ensino Médio. Acta Colombiana de Psicología, 23(1), 254-263. http://doi.org/10.14718/ACP.2020.23.1.12
Sánchez, I. (2021). Impacto psicológico de la COVID-19 en niños y adolescentes. Medisan, 25(1), 123-141

Vásquez, A., Indacochea, F., Forty, J., y Chara, J. (2020). Educación virtual en tiempos del covid-19 desde la perspectiva socioeconómica de los estudiantes de la Universidad Estatal del Sur de Manabí del cantón Jipijapa. Polo Del Conocimiento, 5(10), 798-823. https://doi. org/10.23857/pc.v5i10.1852 